\title{
Collective excitations of atomic Bose-Einstein condensates
}

\author{
Mark Edwards* \\ Department of Physics, Georgia Southern University, Statesboro, GA 30460-8031. \\ P. A. Ruprecht and K. Burnett因 \\ Clarendon Laboratory, Department of Physics, University of Oxford, \\ Parks Road, Oxford OX1 3PU, United Kingdom. \\ R. J. Dodd 1 and Charles W. Clark \\ Electron and Optical Physics Division, National Institute of Standards and Technology, \\ Technology Administration, U. S. Department of Commerce, Gaithersburg, MD 20899.
}

(July 16, 2021)

\begin{abstract}
We apply linear-response analysis of the Gross-Pitaevskii equation to obtain the excitation frequencies of a Bose-Einstein condensate confined in a time-averaged orbiting potential trap. Our calculated values are in excellent agreement with those observed in a recent experiment.
\end{abstract}

PACS Numbers: 3.75.Fi, 67.40.Db, 67.90.+Z 
The recent attainment of quantum degeneracy conditions in magnetically trapped alkali vapors [1,2].3] has opened the road to understanding of the many-body physics of BoseEinstein condensates (BECs) in unprecedented detail. For dilute gases, it is believed that the essential physics of the BEC ground state is captured in the Gross-Pitaevski mean-field formalism. Calculations done with the Gross-Pitaevski (GP) equation [4.5 have indeed agreed reasonably well with the few experimental determinations of condensate shapes, sizes, and lifetimes that have been made to date, but it cannot be said that the theory has been subject to stringent tests.

In this paper we report theoretical results for the excitation spectrum of a BEC of trapped ${ }^{87} \mathrm{Rb}$, obtained by computing the response to small mechanical disturbances of a BEC described by the GP equation [6]. These results are compared with those of a recent experiment [7], which has observed the free oscillations of a BEC that is briefly shaken at frequencies near resonance. We believe that this comparison provides the most critical quantitative test of mean-field theory made to date. The agreement between experimental and theoretical results is excellent. This suggests that the GP equation and its variants can adequately describe excited-state as well as ground-state properties, and so should provide a practical framework for explorations of emerging issues of condensate dynamics, finitetemperature phenomena, and atom laser [8] design.

To describe a magnetically trapped atomic gas, we adopt the standard GP equation, which is applicable [9:10] when the condensate fraction of a gaseous system is close to unity. Each atom in the condensate occupies the same orbital $\psi_{g}(\mathbf{r})$, which is determined by solution of the nonlinear Schrödinger equation,

$$
\left[H_{0}+N_{0} U_{0}\left|\psi_{g}(\mathbf{r})\right|^{2}\right] \psi_{g}(\mathbf{r})=\mu \psi_{g}(\mathbf{r})
$$

where $H_{0}=-\frac{\hbar^{2}}{2 m} \nabla^{2}+V_{\text {trap }}(\mathbf{r})$ is the Hamiltonian for an isolated atom in the trap, $N_{0}$ is the number of atoms in the condensate, $U_{0}$ represents the interaction between condensate atoms, and the eigenvalue $\mu$ is the chemical potential.

In most current trap designs, $V_{\text {trap }}$ can be described by the anisotropic harmonic oscillator 
potential, $V_{\text {trap }}(\mathbf{r})=\frac{1}{2} m\left(\omega_{x}^{2} x^{2}+\omega_{y}^{2} y^{2}+\omega_{z}^{2} z^{2}\right)$, where $m$ is the atomic mass and $\omega_{i}=2 \pi \nu_{i}$ is the angular frequency of oscillation along the axis $i$. The time-averaged orbiting potential (TOP) trap [1, [1] treated here is cylindrically symmetric; its potential is given by $\omega_{x}=\omega_{y}=$ $\omega_{\perp}$ and $\omega_{z}=\sqrt{8} \omega_{\perp}$. The results presented below correspond to $\nu_{z}=\omega_{z} / 2 \pi=210 \mathrm{~Hz}$. The parameter $U_{0}$ expresses the interaction between two atoms as $U_{0}=4 \pi \hbar^{2} a / m$, where $a$ is the scattering length, which characterizes the zero-energy behavior of the $s$-wave phase shift in collisions between two atoms. The scattering length $a$ is the only piece of atomic collision data used as input to our calculations. The present results are given in terms of the most recent [15] experimental value, $a=110 a_{0}$, where $a_{0}$ is the Bohr radius; our calculations were actually carried out with a previously published [16] value of $a=100 a_{0}$, but since Eq. 11 obeys a scaling law involving $N_{0}, \nu_{\perp}$, and $a$ (see below), we can rescale our results to compare quantitatively with experiment. It should be noted that for the alkali atoms in current BEC studies, the experimental determination of $a$ requires extensive spectroscopic analysis, and present values are accompanied by substantial uncertainties [12].

Equation 1 has previously been solved by several independent methods [4,5] to describe the BEC ground state. Here we investigate the response of the ground state to an oscillatory perturbation at angular frequency $\omega_{p}$. The associated time-dependent GP equation takes the form:

$$
i \hbar \frac{\partial \Psi}{\partial t}=\left[H_{0}+U_{0}|\Psi(\mathbf{r}, t)|^{2}+f_{+}(\mathbf{r}) e^{-i \omega_{p} t}+f_{-}(\mathbf{r}) e^{i \omega_{p} t}\right] \Psi(\mathbf{r}, t)
$$

where $f_{ \pm}(\mathbf{r})$ are the spatially-dependent amplitudes of the perturbation. We solve this equation in the linear-response limit. The details of this approach are described elsewhere [13], and we simply state the central results here. By using the Ansatz

$$
\Psi(\mathbf{r}, t)=e^{-i \mu t / \hbar}\left[N_{0}^{\frac{1}{2}} \psi_{g}(\mathbf{r})+u(\mathbf{r}) e^{-i \omega_{p} t}+v(\mathbf{r}) e^{i \omega_{p} t}\right]
$$

we obtain the linear-response equations,

$$
\left[\mathcal{L}-\hbar \omega_{p}\right] u(\mathbf{r})+N_{0} U_{0}\left[\psi_{g}(\mathbf{r})\right]^{2} v(\mathbf{r})=-f_{+}(\mathbf{r}) \psi_{g}(\mathbf{r})
$$




$$
\left.N_{0} U_{0}\left[\psi_{g}^{*}(\mathbf{r})\right]^{2} u(\mathbf{r})+\left[\mathcal{L}-\hbar \omega_{p}\right)\right] v(\mathbf{r})=-f_{-}(\mathbf{r}) \psi_{g}(\mathbf{r})
$$

where $\mathcal{L}=H_{0}-\mu+2 U_{0} N_{0}\left|\psi_{g}(\mathbf{r})\right|^{2}$.

This pair of equations can be solved by expansion in terms of the GP normal-mode equations,

$$
\begin{aligned}
& {\left[\mathcal{L}-\hbar \omega_{\lambda}\right] u_{\lambda}(\mathbf{r})+N_{0} U_{0}\left[\psi_{g}(\mathbf{r})\right]^{2} v_{\lambda}(\mathbf{r})=0,} \\
& N_{0} U_{0}\left[\psi_{g}^{*}(\mathbf{r})\right]^{2} u_{\lambda}(\mathbf{r})+\left[\mathcal{L}+\hbar \omega_{\lambda}\right] v_{\lambda}(\mathbf{r})=0,
\end{aligned}
$$

where $\omega_{\lambda}$ is an eigenvalue and $u_{\lambda}(\mathbf{r}), v_{\lambda}(\mathbf{r})$ are corresponding eigenfunctions. For a homogeneous $\operatorname{BEC}\left(\right.$ i.e. $\left.V_{\text {trap }}(\mathbf{r})=0\right), \omega_{\lambda}$ would be a quasiparticle excitation frequency with a continuous spectrum, corresponding to travelling sound waves. In a trap, however, the elementary excitations of the BEC remained confined, so $u_{\lambda}(\mathbf{r})$ and $v_{\lambda}(\mathbf{r})$ must be squareintegrable, and the spectrum of $\omega_{\lambda}$ is thus discrete. The solution of eq. 3 can then be obtained in the ususal way by a superposition of eigenfunctions:

$$
\left(\begin{array}{c}
u(\mathbf{r}) \\
v(\mathbf{r})
\end{array}\right)=-\frac{1}{\hbar} \sum_{\lambda} \frac{g_{\lambda}}{\omega_{\lambda}-\omega_{p}}\left(\begin{array}{c}
u_{\lambda}(\mathbf{r}) \\
v_{\lambda}(\mathbf{r})
\end{array}\right)
$$

where

$$
g_{\lambda}=\int d^{3} r\left[u_{\lambda}^{*}(\mathbf{r}) f_{+}(\mathbf{r})+v_{\lambda}^{*}(\mathbf{r}) f_{-}(\mathbf{r})\right] \psi_{g}(\mathbf{r})
$$

The response diverges when $\omega_{p}=\omega_{\lambda}$, i.e. when the driving frequency is equal to a natural excitation frequency. In practice, of course, this divergence will be eliminated by damping effects, a subject of ongoing work that will not be discussed here. To the extent that mechanisms of damping and their associated frequency shifts can be neglected, it is apparent that free oscillation of the condensate after a transient disturbance will occur at the frequencies $\omega_{\lambda}$.

As we have discussed elsewhere [13], there is a straightforward connection between the resonant oscillation frequencies $\omega_{\lambda}$ and the the quasi-particle mode frequencies that are 
encountered in the standard Bogoliubov approximation [10]. Stated simply, Eqs. (6) and (7) are identical to the equations that define the quasi-particle modes and frequencies within the Bogoliubov approximation. Thus an experiment that measures the free oscillatory response of a shaken BEC provides a direct observation of the quasi-particle spectrum. In particular, by shaking the BEC at a frequency near one of the resonances $\omega_{\lambda}$, one can produce a response that is dominated by the $\omega_{\lambda}$. This is the approach that has been taken by the first such experiment reported [7].

We have solved numerically the system of equations consisting of Eqs. (1), (6), and (匹) under the conditions of that experiment. The solution was accomplished in two steps. First, Eq. (1) was solved by expanding the solution $\psi_{g}(\mathbf{r})$ in a basis set consisting of a finite number of trap eigenfunctions. The details of the numerical method have been recounted elsewhere [5]. Equations (6), and (7) were then solved by expanding $u_{\lambda}(\mathbf{r})$ and $v_{\lambda}(\mathbf{r})$ in the same basis set. These expansions convert Eqs. (6), and (7) into a generalized matrix eigenvalue problem that can be solved by standard numerical techniques [13]. The error in the solutions was assessed by increasing the basis-set size until the mode frequencies converged to at least three figures.

Figure 1 shows our results for the lowest three excitation frequencies (in units of the trap frequency $\left.\nu_{\perp}^{(t)}\right)$, as a function of $N_{0}$. A simple scaling law faciltiates comparison of

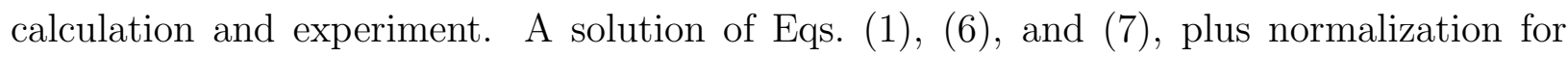
experimental values of the parameters $\left\{N_{0}^{(e)}, a_{e}, \nu_{\perp}^{(e)}, m_{e}\right\}$, will also satisfy the equations for the parameter set $\left\{N_{0}^{(t)}, a_{t}, \nu_{\perp}^{(t)}, m_{t}\right\}$, if the quantity $\gamma=N_{0} a\left(m \nu_{\perp}\right)^{1 / 2}$ is constant. Thus, excitation measurements performed on a BEC with $N_{0}^{(e)}$ atoms in a trap of frequency $\nu_{\perp}^{(e)}$ can be related to the spectrum displayed in Fig. 1 by taking

$$
N_{0}^{(t)}=\left(\frac{a_{e}}{a_{t}}\right)\left(\frac{\nu_{\perp}^{(e)}}{\nu_{\perp}^{(t)}}\right)^{1 / 2} N_{0}^{(e)}
$$

where $\nu_{\perp}^{(t)}=210 / \sqrt{8} \approx 74.25 \mathrm{~Hz}$.

We have used this scaling law to compare our results with those of the recent experiment [7] where excitations of an atomic BEC were observed for the first time. Table [ 
the comparison. No attempt has been made here to account for experimental uncertainties. The ranges presented for the experimental ratio $\left(\nu_{\text {mode }}^{(e)} / \nu_{\perp}^{(e)}\right)$ involve a $1-3 \%$ reduction of the raw data to extrapolate to zero-amplitude driving force [14]. It is fair to say that the agreement is excellent, as the difference between theory and experiment ranges from 2-6\%.

To better understand the nature of these excitations we compare them with the results of Stringari [17] who obtained analytic solutions to the linearized GP equation in the hydrodynamic limit $\left(N_{0} \rightarrow \infty\right)$. The curve labeled " $m=1$ " is a doubly-degenerate dipole excitation that coincides exactly with the first excited state of the bare trap. This is because the lowest dipole mode of an ensemble of identical interacting atoms in an external harmonic potential corresponds to a rigid motion of the center-of-mass, independent of the nature of interatomic forces [17.

The curve labeled " $m=2$ " corresponds to doubly degenerate excitations that correlate to the lowest $m=|2|$ energies of the two-dimensional harmonic oscillator in the noninteracting limit. The quadrupolar nature of these excitations is exhibited in fig. 2, which contains a plot of $u_{\lambda}$ over a region of the $x y$ plane $\left[\right.$ i.e. $\left.u_{\lambda}(x, y, 0)\right]$ for $N_{0}=2000$ atoms. This plot clearly shows the four-peak structure characteristic of quadrupole excitations. The large $-N_{0}$ limit of this mode, $\nu_{\text {mode }} / \nu_{\perp} \rightarrow \sqrt{2}$, is also shown in figure 1 . The fact that the middle curves also seem to be approaching this limit as $N_{0} \rightarrow \infty$ also lends support to their interpretation as quadrupole excitations.

The curve labeled " $\mathrm{m}=0$ " corresponds to a "large $-N_{0}$ " excitation that is a breathing mode in the $x y$ plane. The asymptotic limit is shown in fig. 1 and is given by [17]

$$
\nu_{\text {mode }} / \nu_{\perp} \rightarrow\left(2+\frac{3}{2} \lambda^{2}-\frac{1}{2} \sqrt{9 \lambda^{4}-16 \lambda^{2}+16}\right)^{1 / 2}
$$

where $\lambda=\nu_{z} / \nu_{\perp}=\sqrt{8}$ for the TOP trap. As discussed in ref. [7], the symmetries of the normal modes can be tested by experimental selection rules, and the classifications of the observed modes are found to agreement with those given here.

In conclusion, we have presented excitation spectra that agree well with the data of a recent experiment. We have shown that these data constitute a direct measurement of the 
$T=0$ Bogoliubov spectrum of an atomic BEC. We have also used the large $-N_{0}$ limit and mode shapes to describe the nature of these excitations. The experimental confirmation of these data will have significant implications for understanding of the many-body physics of these dilute, weakly-interacting bosonic systems, and for practical use in future BEC engineering.

\section{ACKNOWLEDGMENTS}

We thank D. S. Jin, J. R. Ensher, M. R. Matthews, C. E. Wieman, and E. A. Cornell for stimulating discussions and for making their experimental data available to us in advance of publication. Work at Oxford was supported with funding from the Rhodes Trust and the U. K. Engineering and Physical Sciences Research Council. M. Edwards acknowledges funding from National Science Foundation grant PHY-9505468 and support from the GSU Foundation. This work was supported in part by the Institute for Theoretical Atomic and Molecular Physics at Harvard University and the Smithsonian Astrophysical Observatory. 


\section{REFERENCES}

* Also: Physics Laboratory, National Institute of Standards and Technology, Technology Administration, U. S. Department of Commerce, Gaithersburg, MD 20899.

$\dagger$ Also: Institute for Physical Science and Technology, University of Maryland at College Park, College Park, MD 20742.

[1] M. H. Anderson, J. R. Ensher, M. R. Matthews, C. E. Wieman, and E. A. Cornell, Science 269, 198 (1995).

[2] C. C. Bradley, C. A. Sackett, J. J. Tollett, and R. G. Hulet, Phys. Rev. Lett. 75, 1687 (1995).

[3] K. B. Davis, M.-O. Mewes, M. R. Andrews, N. J. van Druten, D. S. Durfee, D. M. Kurn, and W. Ketterle, Phys. Rev. Lett. 75, 3969 (1995).

[4] M. Holland and J. Cooper, Phys. Rev. A 53, R1954 (1996).

[5] M. Edwards, R. J. Dodd, C. W. Clark, P. A. Ruprecht, and K. Burnett, Phys. Rev. A 53, R1950 (1996).

[6] We have recently become aware of a similar calculation: L. You, W. Hoston, M. Lewenstein, and K. Huang, (preprint). This paper give no indication of how the excitation frequencies could be measured, however.

[7] D. S. Jin, J. R. Ensher, M. R. Matthews, C. E. Wieman, and E. A. Cornell, (submitted to Phys. Rev. Lett. )

[8] M. Holland, K. Burnett, C. Gardiner, J. I. Cirac, and P. Zoller, Phys. Rev. A (to be published).

[9] See, e.g. A. Griffin, Phys. Rev. B 53, 9341 (1996).

[10] A. L. Fetter, Ann. of Phys., 70, 67 (1972); A. L. Fetter, Phys. Rev. A (to be published). 
[11] W. Petrich, M. H. Anderson, J. R. Anderson, J. R. Ensher, and E. A. Cornell, Phys. Rev. Lett. 74, 3352 (1995).

[12] E. Tiesinga, C. J. Williams, P. S. Julienne, K. M. Jones, P. D. Lett, and W. D. Phillips, J. Res. Nat. Inst. Standards and Technology 101 (1996 in the press)

[13] M. Edwards, P. A. Ruprecht, K. Burnett, and C. W. Clark, (submitted to Phys. Rev. A).

[14] E. A. Cornell, private communication.

[15] D. Heinzen, private communication.

[16] J. R. Gardner, R. A. Cline, J. D. Miller, D. J. Heinzen, H. M. J. M. Boesten and B. J. Verhaar, Phys. Rev. Lett. 74, 3764 (1995).

[17] S. Stringari, preprint (cond. mat. 9603126). 


\section{FIGURES}

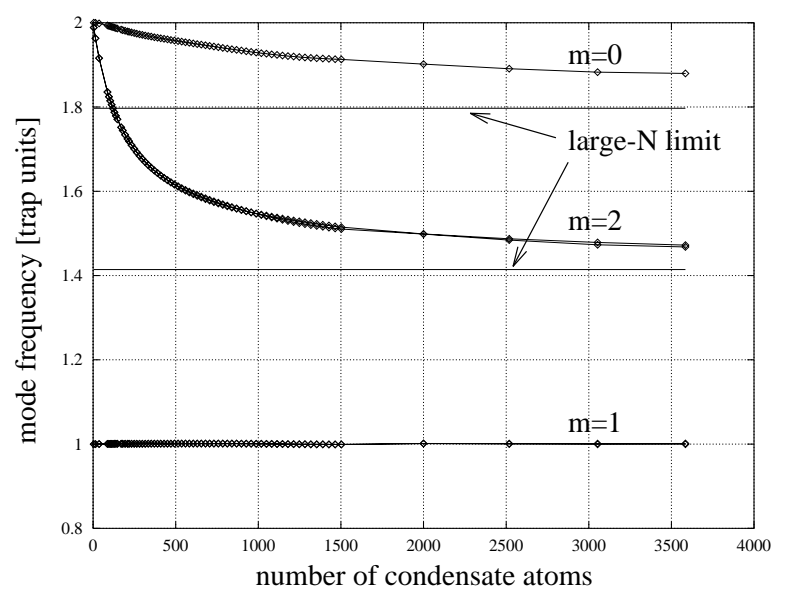

Figure 1, "Collective excitations of atomic Bose-Einstein condensates",

M. Edwards, R.J. Dodd, C.W. Clark, P.A. Ruprecht, and K. Burnett

FIG. 1. The lowest three calculated excitation frequencies, in units of the perpendicular trap frequency $\nu_{\perp}^{(t)}$, of the JILA TOP-trap condensate, it vs. the number of condensate atoms, $N_{0}^{(t)}$.

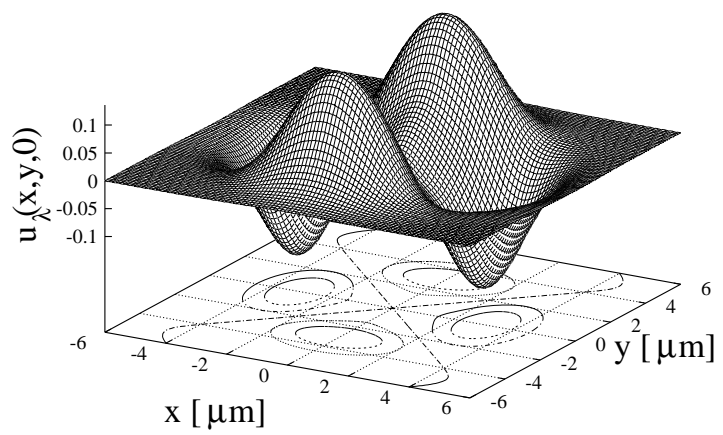

Figure 2, "Collective excitations of atomic Bose-Einstein condensates,"

M. Edwards, R.J. Dodd, C.W. Clark, P.A. Ruprecht, and K. Burnett

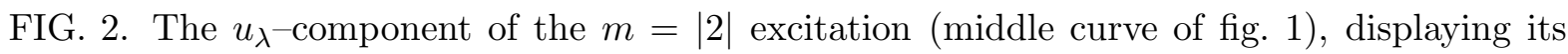
quadrupolar shape. 


\section{TABLES}

TABLE I. This table presents the comparison with excitation data obtained in the experiment of ref. [4].

\begin{tabular}{lccccccc}
\hline \hline mode & $\nu_{\perp}^{(e)}$ & $\nu_{\text {mode }}^{(e)}$ & $N_{0}^{(e)}$ & $\frac{\nu_{\text {mode }}^{(e)}}{\nu_{\perp}^{(e)}}$ & $N_{0}^{(t)}$ & $\frac{\nu_{\text {mode }}^{(t)}}{\nu_{\perp}^{(t)}}$ & $\%$ diff. \\
\hline$m=0$ & 43.2 & 82 & 3420 & $1.84-1.88$ & 2870 & 1.89 & 2.7 \\
$m=0$ & 132 & 244.7 & 2400 & $1.79-1.83$ & 3520 & 1.88 & 5.0 \\
$m=2$ & 43.2 & 62.5 & 2800 & $1.41-1.44$ & 2350 & 1.49 & 5.7 \\
$m=2$ & 132 & 188.5 & 2200 & $1.39-1.42$ & 3230 & 1.47 & 5.8 \\
\hline \hline
\end{tabular}

